\title{
EL PROCESO DE ENSENANANA- APRENDIZAJE UN RETO EN EL CAMBIO EDUCATIVO
}

\author{
AUTORES: Raquel Vera Velázquez ${ }^{1}$ \\ Kirenia Maldonado Zúñiga ${ }^{2}$ \\ Yoiler Batista Garcet ${ }^{3}$ \\ Alfredo Lesvel Castro Landin ${ }^{4}$
}

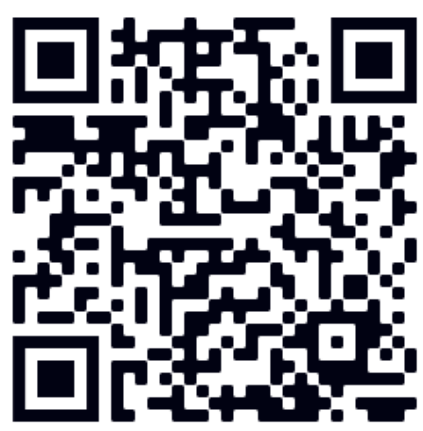

\section{DIRECCIÓN PARA CORRESPONDENCIA:raquelita2015vera@gmail.com}

Fecha de recepción: 11/06/2019

Fecha de aceptación: 27/11/2019

\section{RESUMEN}

Teniendo en cuenta que los tiempos presentes y futuros reclaman de un estudiante capaz de poner en práctica estrategias adecuadas para aprender que sean de un aprendizaje guiado por el docente como facilitador, obteniendo como resultado decisiones conscientes, intencionales y contextualizadas de cómo proceder para lograr determinado objetivo de aprendizaje, se aborda en este trabajo el tema del importante papel del profesor en la enseñanza de estrategias de enseñanza-aprendizaje, para analizar cómo a través del propio desarrollo de su asignatura y mediante el empleo de diversos métodos puede alcanzar este fin. Para ello se analizaron documentos, se hizo una revisión bibliográfica, la exposición de algunas experiencias, valoraciones y reflexiones personales a través de una encuesta realizada a 53 docentes de la Universidad Estatal del Sur de Manabí, a las carreras Agropecuaria, Laboratorio Clínico y Computación y Redes, resultados que pusieron en evidencia una gran coincidencia entre las concepciones expuestas acerca de los métodos que debe utilizar el profesor para contribuir a la formación de estrategias de aprendizaje en los estudiantes. En ellas se destacan la posibilidad de seguir las recomendaciones que se derivan de las reflexiones y vivencias de los expertos; el uso de preguntas para guiar al estudiante en su razonamiento, así como otras formas de ayuda, digamos, la modelación y el adiestramiento; la reflexión a posteriori sobre el proceso seguido para alcanzar un fin y su comparación con los resultados obtenidos y además la realización de actividades cooperativas para resolver las dificultades arrojadas en el estudio realizado.

\footnotetext{
${ }^{1}$ 1Máster en Ciencias de la Educación. Universidad de Ciencias Pedagógicas, Las Tunas Cuba.raquelita2015vera@gmail.com

${ }^{2}$ Máster en Ciencias de la Educación. Universidad de Ciencias Pedagógicas, Holguín Cuba.kmzmaldonado@gmail.com

${ }^{3}$ Máster en Ciencias de la Educación. Universidad de Ciencias Pedagógicas, Las Tunas yoiler.batista@unesum.edu.ec

${ }^{4}$ Máster en Ciencias Agrícolas. Universidad Camilo Cienfuegos, Matanzas.alfredolandin.55@gmail.com
} 
PALABRAS CLAVE: estrategias de aprendizaje; métodos de enseñanza; estrategias de enseñanza, metodologías de aprendizaje y estilos del profesor.

\section{THE TEACHING PROCESS - LEARNING A CHALLENGE IN EDUCATIONAL CHANGE}

\section{ABSTRACT}

Bearing in mind that present and future times demand a student capable of implementing appropriate strategies to learn that are from teacher-guided learning as a facilitator, resulting in conscious, intentional and contextualized decisions on how to proceed to achieve a specific objective. In this work, the topic of the important role of the teacher in teaching teaching-learning strategies is addressed in this work, to analyze how through the development of his subject and through the use of various methods he can achieve this goal. For this, documents were analyzed, a bibliographic review was made, the exposition of some experiences, assessments and personal reflections through a survey of 53 teachers from the State University of the South of Manabí, agricultural careers, Clinical Laboratory and Computation and Networks, results that showed a great coincidence between the exposed conceptions about the methods that the teacher should use to contribute to the formation of learning strategies in the students. They highlight the possibility of following the recommendations that derive from the reflections and experiences of the experts; the use of questions to guide the student in his reasoning, as well as other forms of help, say, modeling and training; a posteriori reflection on the process followed to achieve an end and its comparison with the results obtained and also the realization of cooperative activities to solve the difficulties thrown in the study.

KEYWORDS: learning strategies; teaching methods; teaching strategies, learning methodologies and teacher styles.

\section{INTRODUCCIÓN}

La didáctica, rama fundamental de la pedagogía, tiene como parte de los requisitos que le confieren su carácter de ciencia, un objeto de estudio bien delimitado: el proceso de enseñanza aprendizaje.

Por increíble que parezca, todavía existen profesionales de la educación que ignoran que este es el objeto de estudio de la didáctica actual, y con ello el carácter renovador que la misma encierra para tributar el cambio educativo que reclama el nuevo milenio.

La necesidad de preparar a las generaciones presentes y futuras para asumir un papel más activo y creador en el proceso de construcción de su propio desarrollo, en beneficio de la sociedad, es una preocupación actual de todos los que de una forma u otra asumimos una parte de la responsabilidad en el logro de ese propósito. Esto equivale a decir que hay que lograr que el estudiante sea capaz de aprender a aprender, lo que significa prepararlo para que pueda dirigir su propio aprendizaje poniendo en práctica estrategias apropiadas que sean el resultado de decisiones conscientes, intencionales y contextualizadas de cómo proceder para lograr determinado objetivo relacionado con su aprendizaje. En ese empeño los docentes tienen una enorme responsabilidad pues son los que a través de la enseñanza pueden lograr que el estudiante aprenda cómo se aprende. Ellos tienen la posibilidad de conducir el complejo proceso de desarrollo de estrategias de aprendizaje mediante sus estrategias de enseñanza.

102 UNESUM-Ciencias.Publicación cuatrimestral. Vol. 3, Año 2019, No. 2 (Mayo - Agosto) 
UNESUM-Ciencias: Revista Científica Multidisciplinaria ISSN 2602-8166

EL PROCESO DE ENSEÑANZA- APRENDIZAJE UN RETO EN EL CAMBIO EDUCATIVO

Estrategias de Aprendizaje.

El estudio de los trabajos acerca del tema de las estrategias de aprendizaje permite comprender que este es un asunto de enorme importancia en la actualidad educacional y que son diversas las acepciones que se le atribuyen a este concepto, tanto en sentido estrecho como amplio. En esta línea el razonamiento Monereo y Pérez Cabani (1996) plantean:

Así, las estrategias de aprendizaje se han conceptualizado como un conjunto de operaciones mentales y procedimientos de codificación, adquisición, retención y evocación (Rigney, 1978; Weinstein y Mayer, 1986), como planes generales para lograr objetivos instruccionales (Snowman, 1986), como procesos generales de control cognitivo (Poggioli, 1989), como una combinación de habilidades cognitivas que se activan frente a situaciones percibidas como demandas de aprendizaje (Schmeck, 1989), como procesos de mediación cognitiva (Monereo, 1990) o como sistema de autorregulación cognitiva (Zimmerman, 1990). (p.66) (Mar Cornelio, Gulín González et al. 2016).

Este panorama nos permite comprender que a pesar de la relación y la complementación de estas definiciones es preciso, para ganar en coherencia interna, dejar planteado de forma explícita la que guiará el proceso de reflexión en este trabajo (Mar, Véliz et al. 2015). Si tomamos en cuenta que en esas definiciones se habla de procedimientos, objetivos a lograr, situaciones percibidas, mediación cognitiva y autorregulación cognitiva, como elementos esenciales de la actuación estratégica, entonces nos parece adecuado usar la conceptualización propuesta por Monereo y Pérez Cabani (1996) que se define así: "procesos de tomas de decisiones, conscientes e intencionales, en los cuales el estudiante elige y activa, de manera coordinada, aquellos conocimientos declarativos y procedimentales que necesita para cumplimentar una determinada demanda, en función de las condiciones de la situación en que se produce dicha demanda".(p.66)

Comprendido así, entonces el estudiante actúa estratégicamente cuando tiene una finalidad y objetivo consciente y conociendo las características específicas de la situación en que se encuentra, determina y organiza la aplicación de los procedimientos de aprendizaje. Cabe, entonces preguntarnos: ¿Tiene el profesor alguna implicación en el proceso de formación y desarrollo de las estrategias de aprendizaje de sus estudiantes?; ¿Cuál es el papel del profesor en este sentido?

Si comprendemos el carácter bilateral del proceso de enseñanza-aprendizaje será fácil entender que el profesor al guiar el desarrollo de sus estudiantes a través de ese proceso no solo lo hace proporcionándoles la adquisición de los conocimientos necesarios, sino también, experiencias, normas, valores, la técnica, los modos de actuación, los hábitos y las habilidades. El accionar del profesor -su estrategia de enseñanza- puede servir de modelo a los estudiantes para su actuación estratégica (Mar and Gulín 2018) (Cornelio and Gulín 2018). No obstante, no podemos desconocer que el estudiante en este nivel ya ha ido adquiriendo recursos estratégicos y que se enfrenta a las nuevas situaciones de aprendizaje a partir de ese referente o grado de competencia cognitiva alcanzado, lo cual puede entorpecer o facilitar la adquisición de nuevas estrategias implícitas o explícitas en la actividad del profesor. En este sentido, en estudios realizados por Monereo y Pérez Cabaní (1996) se constató que "determinadas estrategias de enseñanza favorecen o dificultan la utilización de determinadas estrategias de aprendizaje" (p.78).

El profesor por su preparación y experiencia está llamado a mediar en el proceso de construcción del conocimiento por parte del estudiante guiando y facilitando el aprendizaje. Para ello el profesor debe desde la propia planificación curricular concebir no solo los conocimientos sino 
también otros elementos didácticos entre los que se debe encontrar las habilidades y los hábitos que se refiere a los distintos modos de asimilación de la actividad- y que constituyen recursos estratégicos importantes por sí mismos o como elementos dentro de un sistema estratégico o estrategia mayor. La selección consciente por el profesor de un conjunto de acciones encaminadas a alcanzar determinado objetivo se convierte de hecho en un modelo para el estudiante que puede reflexionar sobre su forma personal de aprender y tomar decisiones al respecto.

El profesor si quiere enseñar a aprender, enseñar estrategias de aprendizaje a sus estudiantes, está llamado en resumen a (Mar-Cornelio, et al. 2019) (Mar, Santana et al. 2019):

Enseñarles a reflexionar sobre su propia manera de aprender, ayudándolos a analizar las operaciones y decisiones mentales que realizan, con el fin de mejorar los procesos cognitivos que ponen en acción. (Análisis metacognitivo).

Implícitamente supone que, como docentes, reflexionemos sobre nuestra propia manera de planificar, presentar y evaluar los distintos contenidos de la materia que enseñamos.

Enseñarles a conocerse mejor como "aprendices", a identificar el formato y origen de sus dificultades, habilidades y preferencias en el momento de aprender, con el triple objetivo de, por un lado, tratar de anticipar y compensar sus lagunas y carencias durante el aprendizaje, en segundo lugar, conseguir un mejor ajuste entre sus expectativas de éxito y el rendimiento obtenido y, por último, favorecer la adaptación de las actividades y ejercicios presentados a sus propias características; en definitiva ayudarles a construir su propia identidad cognitiva. Con respecto a nuestro rol de profesores, reconstruir conscientemente nuestros significados como "enseñantes", con respecto a qué es lo que debe o no enseñarse y cómo debe hacerse para que el estudiante aprenda de forma consistente; supone en último término un reconocimientos a nuestras habilidades y carencias como profesores (estilo de enseñanza) que nos sitúe en la tesitura de emprender cambios que mejoren nuestra actuación profesional.

Enseñarles a dialogar internamente, activando sus conocimientos previos sobre el material a tratar, y relacionándolos de manera sustancial con cada nueva información. Asimismo mostrar, en ocasiones, nuestros propios diálogos internos con el fin de ilustrar y modelar la forma cómo los contenidos específicos de nuestra materia afectan al razonamiento.

Enseñarles a ser intencionales y propositivos cuando aprendan y a entrar en las intenciones de los demás, en especial de sus profesores, para ajustarse mejor a sus intenciones y demandas. Consecuentemente como profesores deberíamos facilitarles la tarea, explicitando nuestras intenciones educativas, lo que conlleva un proceso preliminar de autorreflexión con el fin de clarificarlas.

Enseñarles que no deben estudiar para aprobar sino para aprender, que únicamente se aprende con profundidad cuando lo aprendido es fruto de un esfuerzo de comprensión y, sobre todo, mostrarles que aprender así es a la larga más rentable porque es más duradero y funcional. En calidad de profesores podríamos, recordando la máxima de Séneca "los hombres aprenden mientras enseñan", aprender a enseñar mejor esforzándonos en comprender los motivos que nos impulsan a tomar determinadas decisiones mientras realizamos una sesión de clase.

Por último, si se nos permite una transgresión morfológica, enseñarles conciencia, en un doble sentido; en relación a los estudiantes, enseñarles a actuar de un modo científico en su aprendizaje, convirtiendo las ideas en hipótesis, comprobando la validez de esas ideas mediante su

104 UNESUM-Ciencias.Publicación cuatrimestral. Vol. 3, Año 2019, No. 2 (Mayo - Agosto) 
experimentación o su confrontación con otras ideas, interpretando los resultados obtenidos y reformulando, en su caso, las premisas de partida.

En relación a nuestra intervención como profesionales, tomar en consideración los conocimientos que han producido la investigación educativa sobre los procesos de enseñanza-aprendizaje para cortejarlos con nuestra práctica docente y reelaborar nuestras ideas sobre cómo debemos enseñar para que nuestros estudiantes "aprendan a aprender" (MONEREO, 1995, pp.8-9), (Mar Cornelio, et al. 2020). En fin como señala Monereo (1995), "enseñar estrategias implica enseñar al estudiante a decidir conscientemente los actos que realizará, enseñarles a modificar conscientemente su actuación cuando se oriente hacia el objetivo buscado y enseñarles a evaluar conscientemente el proceso de aprendizaje o de resolución seguido" (p.8). Estas consideraciones nos llevan a ratificar que el verdadero aprendizaje es aquel que se adquiere de forma activa y consciente, mediante un sistema de acciones mentales y prácticas emprendidas por el estudiante y que tienen como precedente la orientación y guía del profesor. La ayuda que el profesor brinda al estudiante variará cuantitativa y cualitativamente a lo largo del proceso de enseñanzaaprendizaje, es decir, como plantea Pérez Cabaní (1995). "la ayuda requerida en cada momento del proceso será variable en forma y cantidad. A veces, el ajuste de la ayuda pedagógica se conseguirá proporcionando al estudiante una información organizada y estructurada; en otras ocasiones, ofreciendo modelos de acción a imitar, en otras, formulando indicaciones y sugerencias más o menos detalladas para abordar los trabajos, o en otros casos, permitiéndole que escoja y desarrolle de forma totalmente autónoma la actividad de aprendizaje".(p.2)

Por las razones mencionadas la presente investigación tuvo como objetivos: i) determinar las dificultades en el proceso de enseñanza-aprendizaje en las carreras Agropecuaria, Laboratorio Clínico y Computación y Redes de la Universidad Estatal del Sur de Manabí y ii) proponer algunas estrategias para resolver las dificultades existentes.

\section{DESARROLLO}

\section{MATERIALES Y MÉTODOS}

Sitio de estudioLa presente investigación se realizó en el año 2019 en la Carreras de Agropecuaria, Laboratorio Clínico y Computación y Redes de la Universidad Estatal del Sur de Manabí, ubicada en el Campus Los Ángeles, vía Noboa Km 1 1⁄2 s/n, en Jipijapa, Manabí

Metodología

Cuestionario

Se elaboró una encuesta partiendo de los planteamientos de los diferentes autores consultados y considerando los criterios orientadores sobre el proceso de enseñanza-aprendizaje y su didáctica derivados del marco teórico, de 8 preguntas cerradas y para su construcción se analizaron escalas previamente validadas en diferentes investigaciones, analizando los temas sobre el proceso de enseñanza- aprendizaje:

1-Funciones didácticas de la clase.

2-Formas de enseñanza.

3-Formas de aprendizaje.

4-Los métodos de enseñanza en función de la relación enseñanza -aprendizaje.

5-Las metodologías de aprendizaje. 
Se aplicó la encuesta a docentes de las carreras antes mencionadas sobre las estrategias de aprendizaje aplicadas en clases teniendo en cuenta las funciones didácticas de la clases, los métodos y metodologías utilizadas en el desarrollo del proceso de enseñanza- aprendizaje y los contenidos de las asignaturas en las carrerasde agropecuaria y laboratorio clínico de la Universidad Estatal del Sur de Manabí como se muestra en la siguiente tabla \# 1.

\begin{tabular}{|l|l|}
\hline Carreras & Docentes \\
\hline Agropecuaria & 15 \\
\hline Laboratorio Clínico & 18 \\
\hline Computación y Redes & 20 \\
\hline Total & 53 \\
\hline
\end{tabular}

Todo esto nos lleva a considerar que el profesor podrá contribuir al desarrollo de estrategias de aprendizaje en sus estudiantes siempre y cuando tenga mayores conocimientos de la didáctica y su proceso de enseñanza- aprendizaje.

El instrumento aplicado estuvo dirigido a medir el nivel de conocimiento que tienen los docentes sobre la didáctica y su metodología de enseñanza- aprendizaje.

La administración del cuestionario se realizó en las aulas de las carreras Agropecuaria, Laboratorio Clínico y Computación y Redes por los propios investigadores, durante el desarrollo del IX Seminario Científico Metodológico. Antes de entregar el cuestionario, el evaluador proporcionó las instrucciones del llenado y la finalidad de la aplicación del cuestionario. No se limitó el tiempo de respuesta.

Análisis estadísticos

El análisis estadístico se realizó a través del uso de la estadística descriptiva, para lo que se determinaron las frecuencias de respuesta para cada pregunta.

Para entender con mejor precisión el dominio de los docentes respecto a los elementos del proceso de enseñanza- aprendizaje, se analizó las frecuencias de respuesta a través de un diseño de bloques completamente aleatorizados: Previo al análisis de varianza, se determinó la distribución de normalidad y homogeneidad de varianzas. Las medias de frecuencia se ajustaron a una curva normal, para su respetivo análisis.

ANÁLISIS Y RESULTADOS

\begin{tabular}{|l|l|l|}
\hline Carreras & Frecuencia & $\%$ \\
\hline Agropecuaria & 8 & 53,3 \\
\hline Laboratorio Clínico & 10 & 55,5 \\
\hline Computación y Redes & 10 & 50 \\
\hline Total & 38 & 71 \\
\hline
\end{tabular}

106 UNESUM-Ciencias.Publicación cuatrimestral. Vol. 3, Año 2019, No. 2 (Mayo - Agosto) 
UNESUM-Ciencias: Revista Científica Multidisciplinaria ISSN 2602-8166

EL PROCESO DE ENSEÑANZA- APRENDIZAJE UN RETO EN EL CAMBIO EDUCATIVO

RESULTADOS POR CARRERA

Frecuencias

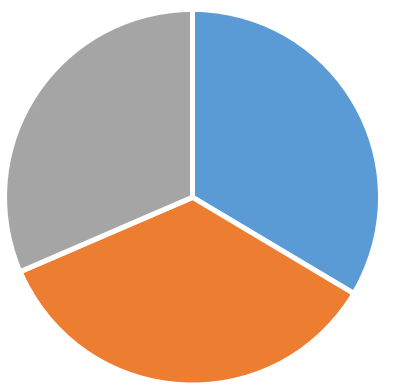

- Agropecuaria - Laboratorio Clínico - Computación y Redes

DISTRIBUCIÓN DE LAS TEMÁTICAS POR ELEMENTOS DEL CONOCIMIENTO.

\begin{tabular}{|l|l|l|}
\hline Categorías temáticas & Frecuencias & Porcentajes \\
\hline 1-Funciones didácticas de la clase. & 35 & 66 \\
\hline 2-Formas de enseñanza. & 33 & 62,2 \\
\hline 3-Formas de aprendizaje. & 35 & 66 \\
\hline $\begin{array}{l}\text { 4-Los métodos de enseñanza en función de } \\
\text { la relación enseñanza -aprendizaje. }\end{array}$ & 36 & 67,9 \\
\hline 5-Las metodologías de aprendizaje & 34 & 64,1 \\
\hline
\end{tabular}

\section{RESULTADOS POR INDICADORES}




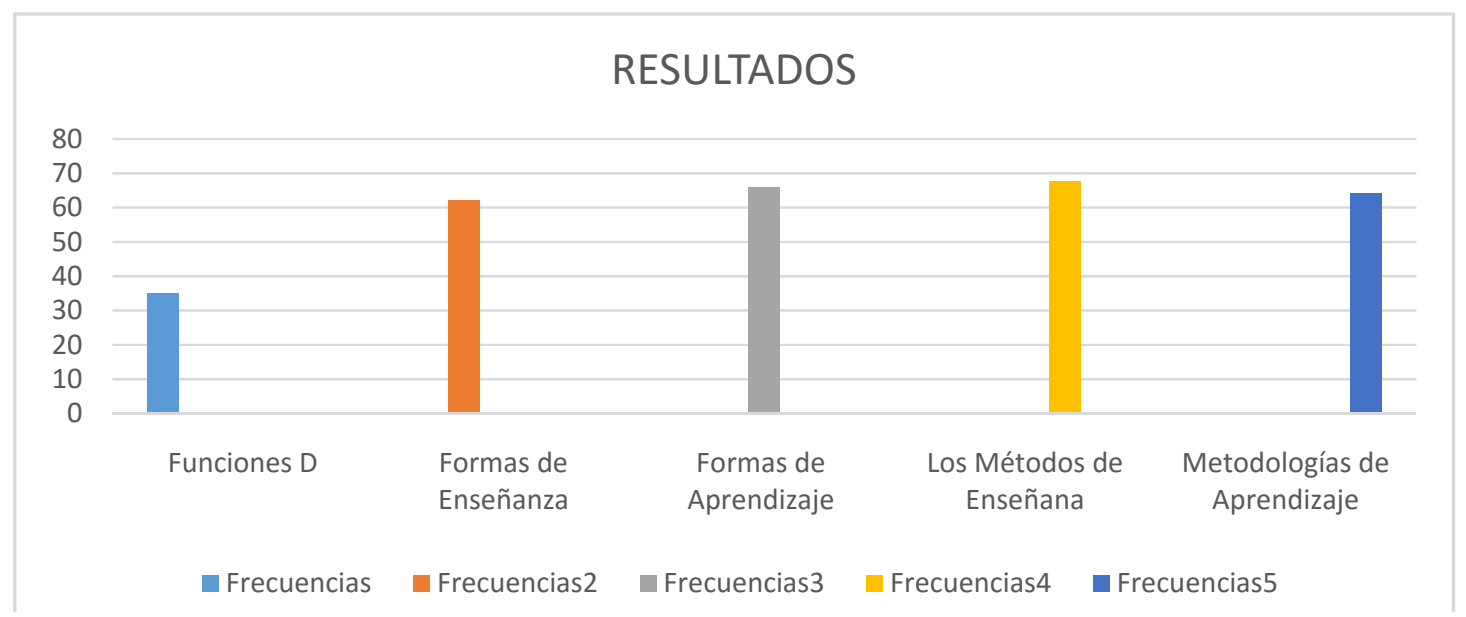

Como podemos observar en el indicador 1 funciones didácticas de la clase solo 35 de los 53 encuestados se plantearon formas de motivación de la clase y demostraron dominio de la estructura didáctica de la clase lo que representa el $66 \%$ de los encuestados.

En el indicador 2 las formas de enseñanza solo 33 de los 53 encuestados demostraron habilidades en las formas de enseñanza lo que representa el $62,2 \%$ de los encuestados.

En el indicador 3 las formas de aprendizaje de los estudiantes 35 fueron capaces de plantearse diferentes formas de como el estudiante aprende utilizando el modelo educativo de la universidad, lo que representa un $66 \%$.

En el indicador 4, los métodos de enseñanza solo 36 demostraron dominio de los diferentes métodos de enseñanza, lo que representa el $67,9 \%$ de los evaluados.

En el indicador 5, las metodologías de aprendizaje utilizando el constructivismo como modelo educativo de la universidad, solo 34 demostraron dominio lo que representa el $64,1 \%$ de los evaluados.

Estos resultados nos dieron evidencias de las dificultades en el dominio de la didáctica y su metodología, por lo que nos planificamos a nivel de carreras realizar cursos de capacitación en estos temas para resolver las dificultades arrojadas en el estudio realizado y obtener mejores resultados en el proceso de enseñanza y aprendizaje de los estudiantes.

\section{CONCLUSIONES}

El profesor mediante el sistema de actividades docentes, laborales e investigativas que dirige en el proceso de educación profesional pone en práctica estrategias de enseñanza que promueven el proceso de formación y desarrollo de las estrategias de aprendizaje de sus alumnos apoyándose en sus referentes cognitivos.

Resulta importante que desde la propia planificación curricular el profesor conciba no solo los conocimientos, llamados declarativos, sino también las habilidades y hábitos, pues constituyen recursos estratégicos importantes por sí mismos o como elementos dentro de otra estrategia. Existe una gran coincidencia entre las concepciones expuestas acerca de los métodos que debe utilizar el profesor para contribuir a la formación de estrategias de aprendizaje en los estudiantes.

108 UNESUM-Ciencias.Publicación cuatrimestral. Vol. 3, Año 2019, No. 2 (Mayo - Agosto) 
En ellas se destacan la posibilidad de seguir las recomendaciones que se derivan de las reflexiones y vivencias de los expertos; el uso de preguntas para guiar al estudiante en su razonamiento, así como otras formas de ayuda, digamos, la modelación y el adiestramiento; la reflexión a posteriori sobre el proceso seguido para alcanzar un fin y su comparación con los de otras personas y además la realización de actividades cooperativas.

La preparación de la clase debe incluir en su estructuración metodológica las indicaciones necesarias para realizar el análisis de las estrategias de enseñanza y aprendizaje empleadas durante la misma y derivar de ellos los ajustes favorecedores de un mejor rendimiento.

La tarea de desarrollar en los estudiantes estrategias de aprendizaje plantea la necesidad de garantizar que desde la propia formación profesional del profesor éste adquiera la preparación idónea para aprender y enseñar a aprender estratégicamente. Los métodos propuestos para el desarrollo de las estrategias de aprendizaje se apoyan y favorecen la comunicación en el aula entre profesores y estudiantes y entre los estudiantes.

\section{REFERÉNCIAS BIBLIOGRÁFICAS}

Explora Natura . (24 de mayo de 2019). Interdisciplinariedad Educación Ambiental. Obtenido de https:/www.exploranatura.com/interdisciplinariedad-educacion-ambiental-2/

Cornelio, O. M. and J. G. Gulín (2018). "Modelo para la evaluación de habilidades profesionales en un Sistema de Laboratorios a Distancia." Revista Cientifica 3(33): 1.

Arichabala. (2014). Los 10 Mejores Proyectos sobre el cuidado del medio ambiente y el manejo de desechos sólidos. Quito: ODEBRECHT.

Mar-Cornelio, O., I. Santana-Ching and J. González-Gulín (2019). "Sistema de Laboratorios Remotos para la práctica de Ingeniería de Control." Revista científica(36): 356-366.

Cadmea, M., \& et al. (2015). La Educación Ambiental en la Educación Superior. Quito: Facultad de Ingenieria Ambiental y recursos naturales.

Mar, O., Y. Z. Véliz, M. d. R. C. Felipe and M. L. Vázquez (2015). "Motor de inferencia decisional en sistema informático para la evaluación del desempeño." Revista Cubana de Ciencias Informáticas 9(4): 16-29.

Carranza, R. (2005). El constructivismo como estrategia educativa:formación profesional . Ensayo, 5-12.

Mar Cornelio, O., J. Gulín González, I. Santana Ching and L. Rozhnova (2016). "Sistema de Laboratorios a Distancia para la práctica de Control Automático." Revista Cubana de Ciencias Informáticas 10(4): 171183.

Follari, R. (2001). Estudios culturales, transdisciplinariedad e interdisciplinariedad. Unidad y Praxis.

Mar, O., I. Santana and J. Gulín (2019). "Algorithm to determine and eliminate neutral nodes in Cognitive Neutrosophic Map." Neutrosophic Computing and Machine Learning 8: 4-11.

Martínez , \& Balaguer. (2016). Universidad saludable: una estrategia de promoción de la salud y salud en todas las políticas para crear un entorno de trabajo saludable. Barcelona: Arch Prev Riesgos Labor vol.19 no.3.

Mar Cornelio, O., I. Santana Ching and J. Gulín González (2020). "Operador por selección para la agregación de información en Mapa Cognitivo Difuso." Revista Cubana de Ciencias Informáticas 14(1): 20-39.

Ministerio del Ambiente. (junio de 24 de 2019). Dirección de Información, Seguimiento y Evaluación -DISE. Obtenido de maeduca@ambiente.gob.ec

Mar, O. and J. Gulín (2018). "Model for the evaluation of professional skills in a remote laboratory system." Revista científica 3(33): 332-343.

(C) Universidad Estatal del Sur de Manabí. Jipijapa, Ecuador. 
Plan Nacional de Desarrollo. (2017-2021). Sobre los aprendizajes compartidos: Presencia e iniciativas de las instituciones a favor de la educación ambiental. Quito: Educación para el desarrollo sostenible.

Sampieri. (2018). Metodología de la investigación: las rutas cuantitativa, cualitativa y mixta. Mexico: McGraw Hill Mexico.

110 UNESUM-Ciencias.Publicación cuatrimestral. Vol. 3, Año 2019, No. 2 (Mayo - Agosto) 
UNESUM-Ciencias: Revista Científica Multidisciplinaria ISSN 2602-8166

EL PROCESO DE ENSEÑANZA- APRENDIZAJE UN RETO EN EL CAMBIO EDUCATIVO 